\title{
Alveolar Surfactant and Inflammatory Mediators
}

\author{
M. HALLMAN
}

Alveolar surfactant is a lipid protein complex that prevents atelectasis, decreases work of breathing, and promotes uniform expansion of alveoli allowing efficient gas exchange. In addition, the surfactant protects the alveoli and the small airways against edema and mechanical trauma. Surfactant also contains components that are involved in defense against microbes and xenobiotics. According to present hypothesis non-clonal innate immunity - an ancient non-vertebrate defense system - is important in host defense particularly in transcellular spaces. This system has evolved to function in a natural state of infectious and inflammatory diseases. The present methods of intensive care have been developed in the absence of the knowledge of the function of the host defense system. It is conceivable that the new and effective invasive treatment practices "fool" the innate immunity to trigger an inappropriate response that becomes destructive rather than protective to the host.

The surfactant system is of central importance in pulmonary gas exchange and important in pulmonary innate immunity. Investigation on the roles of inflammatory mediators may reveal new concepts of lung protection during intensive treatment.

\section{Brief description of the surfactant system}

Alveolar surfactant complex is synthesized, secreted and for the most part catabolized in type II alveolar cells. After intracellular transport, storage in lamellar inclusion bodies and exocytosis the surfactant undergoes aggregate transformation in the epithelial lining. Extracellular surfactant participates in dynamic reduction of surface tension in air-liquid interface. Eventually surfactant components are cleared and catabolized by type II cells and pulmonary alveolar macrophages (PAM), and reutilized by type II cells. Alveolar surfactant consists of dipalmitoyl phosphatidylcholine (DPPC), unsaturated phosphatidylcholine, phosphatidylglycerol, phosphatidylinositol and other minor lipids. Four socalled surfactant proteins that comprise up to $10 \%$ of the complex have unique features. Of the hydrophobic surfactant proteins, mature surfactant protein-B induces fast surface adsorption, spreading and surface stability of the phospho- 
lipids. Congenital absence of SP-B gene in man or SP-B gene deletion in mice results in fatal respiratory failure, characterized by abnormal structure of type II cells, abnormal composition and deficient surface activity of surfactant. SP-C is a small, type II cell-specific proteolipid that covalently binds two palmitate molecules, enhancing the surface adsorption of phospholipids. SP-C is expressed in the alveolar cells during early fetal life prior to the other surfactant components. The phenotype of absent SP-C gene expression has thus far not been described. SP-A is a C-type (i.e. collagenous) lectin. It binds to surfactant aggregates and together with SP-B, DPPC, phosphatidylglycerol and $\mathrm{Ca}^{++}$form tubular myelin that is extremely surface active. However, mice lacking SP-A gene expression have no respiratory failure. Instead, they are prone to specific infections introduced to the respiratory tract (group B Streptococcus and respiratory syncytial virus). With the carbohydrate-binding domain, SP-A binds to specific microbes and increases their phagocytosis by alveolar macrophages in vitro. Hence SP-A is a component of the innate immunity. SP-D is another C-type lectin that binds to specific microbes and LPS. Thus far SP-D has not been shown to stimulate phagocytosis of pathogens. Although SP-D has apparently only small effects on surfactant metabolism and does not bind to surfactant complex (it binds phosphatidylinositol, however), deletion of SP-D gene expression resulted in an order of magnitude increase in the surfactant pool size, and to an emphysematous lung condition in adult mice. The roles of SP-D in homeostasis of the lung remain little understood.

\section{Cytokines}

Several cytokines are involved in alveolar growth, morphogenesis or differentiation of surfactant system. Cytokines are small proteins, produced by a variety of cells. They have biologic activity at a very low concentration (pikomolar nanomolar) - apocrine, paracrine or endocrine activity being critically dependent on the concentration, on surface receptors, and on activities of other agonists and antagonists. Inflammatory cells particularly monocyte-macrophages produce very large quantities of proinflammatory cytokines as a result of contact with bacterial products or specific cytokines. Many cytokines have naturally occurring inhibitors (receptor antagonists, soluble receptors or decoy receptors) that moderate and suppress their activity.

In this brief review, several cytokines known to regulate production of surfactant components, are divided into two groups: those that are mainly expressed in the normal lung and those mainly associated with pathological conditions, i.e. during infection or inflammation (i.e. proinflammatory cytokines). The cytokines have a myriad of overlapping, often redundant functions that range from regulation of growth, differentiation, and defense against infection, regulation of immune cells, and many other roles. The effects of cytokines on the surfactant system range from promotion of alveolar cell growth, induction of 
surfactant synthesis to profound suppression of surfactant synthesis, metabolism and alveolar cell damage. Cytokines activate intracellular kinases and specific transcription factors and also induce secondary mediators including nitric oxide (NO), prostaglandins, leukotrienes and others. Nitric oxide and its metabolites have extensive interactions with the surfactant system, and $\mathrm{PGE}_{2}$ influences the expression of a surfactant protein.

\section{Cytokines having roles mainly in normal differentiation}

Epidermal growth factor $(E G F)$ and transforming growth factor- $\alpha(T G F-\alpha)$ are related peptides that both bind to the EGF receptor with different affinities. EGF has been localized in respiratory epithelium [1], and increases also in lung effluent during prenatal development. TGF- $\alpha$ is a proinflammatory cytokine that is expressed in normal fetal airway epithelial cells at all levels. Administration of EGF to fetal rabbits and rhesus monkeys has been shown to increase surfactant phospholipids and SP-A in type II cells, in the amniotic fluid, and to increase the alveolarization and the stability of the premature lung [2-4]. As a result of the androgen-induced delay in the expression of EGF receptor level, female lung may respond more readily than the male lung [5]. Likewise antibody to EGF delays differentiation of surfactant. Mice lacking EGF receptor expression suffer from impaired epithelial development of several organs, including the lung. Some strains of EGF deficient mice die early due to respiratory failure associated with deficient airway branching and lack of alveolar epithelial differentiation [6]. After birth the bronchiolar epithelium in bronchopulmonary dysplasia (BPD; also called chronic lung disease, CLD) shows immunostaining suggesting a role of EGF abnormal regeneration of airways [1].

Transforming growth factor- $\beta$ (TGF- $\beta$ ) belongs to the superfamily of more than 20 dimeric proteins of similar structure [7]. Three TGF- $\beta$ isoforms and several other members of the superfamily are expressed in mammalian tissues. A major role of these highly conserved cytokines is to regulate the formation of extracellular matrix, increasing synthesis and secretion and decreasing the breakdown of a variety of extracellular matrix proteins. Thus an excess of TGF$\beta$ in tissues can lead to an unbalanced deposition of extracellular matrix and contribute to a variety of fibrotic lung disorders. TGF- $\beta 1$, TGF- $\beta 2$, and TGF- $\beta 3$ are expressed in the lung during fetal and postnatal life. The in situ hybridization patterns of these three isoforms are distinct. High levels of TGF- $\beta$ have been reported in epithelial lining fluid from normal humans. TGF- $\beta$ inhibits the expression of SP-A in explants from human lung, and blocks the stimulatory effect of EGF on SP-A expression [3]. A TGF- $\beta$-like activity from lung fibroblasts inhibits surfactant phospholipid production by murine fetal type II cells in vitro. On the contrary, TGF- $\beta 1$ null mice have a tendency to die in utero. Those developing to term have a multifocal fatal inflammatory disease affecting lung and heart [8]. 
Granulocyte-macrophage colony-stimulating factor (GM-CSF) is traditionally involved in regulation of hematopoiesis. GM-CSF that is expressed in alveolar and airway cells, increases in lung effluent more than 10-fold during the last trimester of fetal development [9]. GM-CSF tends to increase in lung effluent among infants developing BPD. Overexpression of GM-CSF by type II alveolar cells increases the alveolar cells (type II and PAM), but does not affect the pool size of surfactant [10]. On the other hand, administration of GM-CSF to the airways of the premature rabbits acutely increased the intracellular transport, exocytosis and extracellular pools of the surfactant phospholipids. In contrast at term, GM-CSF increased the turnover of surfactant phospholipids without affecting the pool size [11]. Mice lacking GM-CSF expression do not have deficiency in peripheral hematopoietic cells. Instead, adult animals develop pulmonary alveolar proteinosis that is characterized by accumulation of excessive quantities of surfactant components in alveolar spaces and in PAM [12], and abnormally slow turnover of surfactant components [13]. GM-CSF appears to have a role in proliferation and differentiation of the alveolar cells, although it does not influence the transcription of SPs.

Keratinocyte growth factor $(K G F)$ is an epithelial cell-specific heparin-binding growth factor. KGF is necessary in embryonic differentiation of type II epithelial phenotype and in the induction SP-C expression [14]. In addition, KGF reduces bleomycin-induced [15] and acid instillation-induced [16] lung injury, increasing type II cell proliferation and SP mRNA expression.

\section{Proinflammatory cytokines}

Tumor necrosis factor- $\alpha(T N F-\alpha)$ includes two structurally and functionally related proteins. TNF- $\alpha$ and TNF- $\beta$ that bind to same receptors (types I and II), have similar activities. TNF- $\alpha$ is mainly produced in monocytes/macrophages and TNF- $\beta$ in lymphoid cells. TNF- $\alpha$ is actively expressed in response to bacterial, viral, parasite, and endogenous (such as free oxygen radicals) toxins. Overproduction of TNF- $\alpha$ in infections, for instance during endotoxin shock, leads to severe toxicity. TNF- $\alpha$ enhances the production of many other cytokines and inflammatory mediators (NO, prostaglandins, acute phase proteins and others). Long term overproduction of TNF may lead to weight loss, anorexia, and excessive catabolism of proteins. TNF- $\alpha$ has also been implicated as a factor in pathogenesis of autoimmune disorders and of graft-versus-host disease. It serves as an immunostimulant and increases the host resistance.

Activated PAMs produce TNF- $\alpha$ and soluble TNF- $\alpha$ receptors [17]. TNF- $\alpha$ levels in lung effluent are elevated in newborn infants with nosocomial pneumonia, not in infants with respiratory distress syndrome in the newborne (RDS). During development of ARDS in trauma, shock or in sepsis the concentrations of TNF- $\alpha$ (but also soluble TNF receptors I and II serving as inhibitors) increase in BAL $[18,19]$. Thus, because of increase in these inhibitors, little biologic 
TNF activity was detected in BAL despite remarkable increase in TNF- $\alpha$ protein. Plasma concentrations of TNF- $\alpha$ are low in IRDS and in ARDS, except in septic shock.

TNF- $\alpha$ decreases the expression of human SP-A and SP-B mRNA and protein in adenocarcinoma cells [20]. TNF- $\alpha$, administered intratracheally to adult mice reduces SP-C and SP-B mRNA and causes inflammatory lung disease [21]. Transgenic mice overexpressing TNF- $\alpha$ by type II alveolar cells under the control of human SP-C gene promoter develop leukocytotic alveolitis and progressive pulmonary fibrosis [22].

Interferon- $\gamma(I F N-\gamma)$ is produced by activated macrophages or CD4 and CD8 $\mathrm{T}$ lymphocytes. It is a potent phagocytotic activator of macrophages, NK cells and neutrophils. IFN- $\gamma$ also enhances lymphocyte functions. As shown in explants from human fetal lung, IFN- $\gamma$ stimulates synthesis of SP-A mRNA and protein without affecting SP-B or SP-C mRNA [23].

Interleukin- 1 (IL-I) family denotes three polypeptides that have rather little structural homology (30-50\%), despite binding to the same cell surface receptors with similar affinity. Of the two IL-1 receptors, only the type I is involved in signaling, whereas type II receptor serves as a decoy receptor that either as a membrane bound or a soluble protein shunts the ligand away from type I receptor. IL- $1 \alpha$ and IL-1 $\beta$ induce signal transduction, whereas IL-1ra serves as an inhibitor or moderator of IL-1 activity by binding to the receptor without causing signal transduction. In healthy organism the expression of IL- $1 \alpha$ and IL- $1 \beta$ are generally low whereas the expression level of IL-1ra is more than one order of magnitude higher [24]. In PAM, IL- $1 \alpha$ and IL-1 $\beta$ are induced, and IL-1ra expression increased in response to microbial products.

IL-1 activity and the individual peptides are increased in BAL from ARDS patients [25, 18] and in airway specimens of infants who develop BPD [26]. In intrauterine infection (IUI) the amniotic fluid contains increased concentrations of endotoxin (LPS) and proinflammatory cytokines [27]. At birth, very premature infants that are born due to IUI have increased IL-1 and several other proinflammatory cytokines in airway specimens. These infants have significantly decreased incidence of RDS, yet they have an increased risk to develop BPD [28]. Premature birth, the main cause of infant mortality and BPD, is the result of IUI in $30-60 \%$ of cases.

Recombinant human IL- $1 \alpha$ given intra-amniotically (1500 or $150 \mathrm{ng}$ per fetus) to immature rabbit fetuses caused a dose-dependent increase in the expression of SP-A and SP-B mRNA and protein, and an increase in the amount of DPPC in BAL, compared to vehicle treated animals in the opposite uterine horn. The rabbits delivered on d. 27.0 of pregnancy revealed strikingly increased dynamic compliance during a brief period of mechanical ventilation and homogenous aeration of the lung. In the controls, the low lung compliance and generalized atelectasis were consistent with severe RDS. IL-1ra (20 $\mu \mathrm{g}$ intra-amniotically) had no effect on expression of the SPs, suggesting no endogenous IL-1 
activity [29]. Intra-amniotic IL-1 $\alpha$ had no apparent toxicity, whereas intrafetal IL-1 $\alpha(150 \mathrm{ng})$ caused fetal death [30]. Intra-amniotic IL-1 $\alpha(125 \mu \mathrm{g})$ to immature ovine fetuses increased surfactant phospholipid in BAL, and increased lung compliance and gas exchange during brief period of mechanical ventilation. There was little evidence of lung inflammation [31].

The following findings indicate that IL- 1 directly accelerates the differentiation of the surfactant system. Intra-amniotic IL- $1 \alpha$ neither increased systemic levels of glucocorticoid nor caused a stress response [31]. IL-1 acutely increased the expression of SP-A and SP-B mRNA and protein as studied using explants from immature lung [32]. These effects on SPs are very similar to those observed after the intra-amniotic IL-1. Taken together, these findings suggest that in premature births due to IUI, IL-1 is responsible for the decreased incidence of RDS.

High activities of proinflammatory cytokines, including IL-1, are associated with ARDS and with the development of BPD in the premature. Consistent with the observed surfactant dysfunction in ARDS and in BPD, IL-1 suppressed rather than induced the expression of SP-C and SP-B in "mature" (i.e. term fetal and postnatal) lung explants. The suppression increased with the increase in IL$1 \alpha$ concentration [32]. The suppression of surfactant proteins by IL- 1 is consistent with previous data indicating that intratracheal IL- $1 \alpha$ given to adult rats results in high permeability lung edema and lung inflammation that was dependent on the dose of IL-1 [33]. These detrimental effects of IL-1 were expectedly reduced by administration of IL-1ra, and also by administration of $\mathrm{N}$-acetylcysteine (an agent that increases cellular GSH levels) and dimethyl sulfoxide, a scavenger of hydroxyl radicals. Similar to TNF- $\alpha$ and LPC, administration of IL-1 protects against oxidant lung injury, when IL-1 is administered prior to the induction of oxidant injury [34].

\section{Towards understanding the function of proinflammatory cytokines}

The primary proinflammatory cytokines IL-1 and TNF have many additive or synergistic interactions. Other cytokines, such as IFN- $\gamma$ and TGF- $\alpha$ may have a further additive effect. Likewise, cytokines with anti-inflammatory properties moderate the effects of proinflammatory cytokines specifically (IL-1ra antagonizing IL-1 or soluble TNF receptors antagonizing TNF- $\alpha$ ) or by antagonizing the action of proinflammatory cytokines (TGF- $\beta$, IL-10, IL-6, IL-4, other cytokines). Other mediators, particularly glucocorticoid, also serve as anti-inflammatory agents.

The response elicited by the cytokines is strictly dependent on the host. In explants from near term fetal or postnatal lung, IL-1 and TNF- $\alpha$ additively inhibited the expression of SP-A, -B and -C. LPS that induces IL- 1 and TNF- $\alpha$ in monocyte/macrophages, strongly inhibited the expression of SPs [35]. Dexam- 
ethasone (Dex $10^{-7}-10^{-9} \mathrm{M}$ ) acutely decreased the inhibitory effect of IL-1 and TNF- $\alpha$ [36]. These findings further imply the important role of proinflammatory cytokines in pathogenesis of surfactant defects in ARDS and in BPD.

In very immature lung in vitro, the effects of proinflammatory cytokines and anti-inflammatory agents on the expression of alveolar surfactant proteins are different. Neither TNF- $\alpha$ nor LPS had a detectable effect on SP-A, -B, or C, whereas IL-1 induced SP-A and -B, and moderately increased SP-C [35]. The switch from the IL-1-triggered induction of SP-A and SP-B to the IL-1-induced suppression of SP-B and SP-C took place at the same stage of fetal development than the onset of TNF- $\alpha$ - and LPS-induced suppression of SPs.

We propose that the alveolar epithelial cells of very immature lung respond to IL-1 from the amniotic fluid rather than to LPS. The inflammatory response of the immature alveolar structure is paradoxical: by accelerating surfactant maturity, IL-1 prevents RDS and prepares the fetus for postnatal survival [36, 28]. In contrast, exposure of the immature lung directly to microbial products neither induces lung maturity not elicits a proper inflammatory response. The fetuses either die in utero or develop fulminant hyperacute pneumonia if delivered prematurely. Lack of IL-1 $\beta$ and SP-A in airway specimens, very low inducible NO synthase and of nitrotyrosine immunoreactivities in PAM are characteristics of these premature infants that present with symptoms of severe RDS and persistence of fetal circulation shortly after birth. The appearance of IL-1 $\beta$, SP-A, inducible NO synthase and nitrotyrosine in PAM during the recovery from hyperacute sepsis further suggests that IL-1 is a critical factor in the host defense of immature lung [37].

The proinflammatory cytokines induce free radicals, proteolytic enzymes, inhibit growth and induce lung fibrosis. They are likely to be involved in progression of respiratory disease to ARDS and in generation of clinical and pathologic characteristics of BPD. The decrease in formation of surfactant, induced by the proinflammatory cytokines (IL-1 and TNF- $\alpha$ in particular) is an ancient host defense mechanism. Surfactant defect causes an atelectatic sequestration of the infectious focus (i.e. localized pneumonia) as part of the alveolar host defense against generalized infection.

New therapies that limit the generalized proinflammatory cytokine response during invasive pulmonary treatment (without causing a generalized suppression of the cytokine response) remain to be discovered. New treatment that turns on the expression of surfactant components may prove to be important in prevention of progressive alveolar disease. 


\section{References}

1. Stahlman MT, Orth DN, Gray ME (1989) Immunocytochemical localization of epidermal growth factor in the developing human respiratory system and in acute and chronic lung disease in the neonate. Lab Invest 60:539-547

2. Sundell HW, Gray ME, Serenius FS et al (1980) Effects of epidermal growth factor on lung maturation in fetal lambs. Am J Pathol 100:707-726

3. Whitsett JA, Weaver TE, Lieberman MA et al (1987) Differential effects of epidermal growth factor and transforming growth factor- $\beta$ on synthesis of $\mathrm{Mr}=35,000$ surfactant-associated protein in fetal lung. J Biol Chem 262:7908-7913

4. Goetzman BW, Read LC, Plopper CG et al (1994) Prenatal exposure to epidermal growth factor attenuates respiratory distress syndrome in rhesus infants. Pediatr Res 35:30-36

5. Klein JM, Nielsen HC (1995) Androgen regulation of epidermal growth factor receptor binding activity during fetal rabbit lung development. J Clin Invest 91:425-431

6. Sibilia M, Wagner EF (1995) Strain-dependent epithelial defects in mice lacking the EGF receptor. Science 269:234-238

7. Roberts AB, Sporn MB (1992) Differential expression of the TGF- $\beta$ isoforms in embryogenesis suggests specific roles in developing and adult tissues. Molec Reprod Devel 32:91-98

8. Kulkarni AB, Ward JM, Yaswen L et al (1995) Transforming growth factor- $\beta 1$ null mice. An animal model for inflammatory disorders. Am J Pathol 146:264-275

9. Bry K, Hallman M, Teramo K et al (1997) Granulocyte-macrophage colony-stimulating factor in amniotic fluid and in airway specimens of newborn infants. Pediatr Res 41:105-109

10. Huffman Reed JA, Rice WR, Zsengeller ZK et al (1997) GM-CSF enhances lung growth and causes alveolar type II epithelial cell hyperplasia in transgenic mice. Am J Physiol 273: L715-L725

11. Uy CC, Bry K, Lappalainen U, Hallman M (1999) Granulocyte-macrophage colony-stimulating factor increases surfactant phospholipid in premature rabbits (in press)

12. Dranoff G, Crawford AD, Sadelain M et al (1994) Involvement of granulocyte-macrophage colony-stimulating factor in pulmonary homeostasis. Science 264:713-716

13. Ikegami M, Ueda T, Hull W et al (1996) Surfactant metabolism in transgenic mice after granulocyte-macrophage colony-stimulating factor ablation. Am J Physiol 270:L650-L658

14. Shannon JM, Gebb SA, Nielsen LD (1999) Induction of alveolar type II cell differentiation in embryonic tracheal epithelium in mesenchyme-free culture. Development 126:1675-1688

15. Deterding RR, Havill AM, Yano T et al (1997) Prevention of bleomycin-induced lung injury in rats by keratinocyte growth factor. Proc Ass Amer Physicians 109:254-268

16. Yano T, Deterding RR, Simonet WS et al (1996) Keratinocyte growth factor reduces lung damage due to acid instillation in rats. Am J Respir Cell Mol Biol 15:433-442

17. Buch C, Gallati H, Pohlandt F, Bartmann P (1994) Increased levels of tumor necrosis factor alpha (TNF-alpha) and interleukin-1 $\beta$ in tracheal aspirates of newborns with pneumonia. Infection 22:238-241

18. Suter PM, Suter S, Girardin E et al (1992) High bronchoalveolar levels of tumor necrosis factor and its inhibitors, interleukin-1, interferon, and elastase, in patients with adult respiratory distress syndrome after trauma, shock, or sepsis. Am Rev Respir Disease 145:1016-1022

19. Parsons PE, Moore FA, Moore EE et al (1992) Studies on the role of tumor necrosis factor in adult respiratory distress syndrome. Am Rev Respir Dis 146:694-700

20. Wispe JR, Clark JC, Warner BB et al (1990) Tumor necrosis factor-alpha inhibits expression of pulmonary surfactant protein. J Clin Invest 86:1954-1960

21. Bachurski CJ, Pryhuber GS, Glasser SW et al (1995) Tumor necrosis factor-alpha inhibits surfactant protein C gene transcription. J Biol Chem 270:19402-19407

22. Miyazaki Y, Araki K, Vesin C et al (1995) Expression of a tumor necrosis factor- $\alpha$ transgene in murine lung causes lymphocytic and fibrosing alveolitis. A mouse model of progressive pulmonary fibrosis. J Clin Invest 96:250-259

23. Ballard PL, Liley HG, Gonzales LW et al (1990) Interferon- $\gamma$ and synthesis of surfactant components by cultured human lung. Am J Respir Cell Mol Biol 2:137-143 
24. Dinarello CA (1991) Interleukin-1 and interleukin-1 antagonism. Blood 77:1627-52

25. Siler TM, Swierkosz JE, Hyers TM et al (1989) Immunoreactive IL-1 in bronchoalveolar lavage fluid of high-risk patients and patients with the adult respiratory distress syndrome. Exp Lung Res 15:881-894

26. Kotecha S, Wilson L, Wangoo A et al (1996) Increase in interleukin (IL)-1a and IL-6 in bronchoalveolar lavage fluid obtained from infants with chronic lung disease of prematurity. Pediatr Res 40:250-256

27. Gomez R, Ghezzi F, Romero R et al (1995) Premature labor and intra-amniotic infection: Clinical aspects and role of the cytokines in diagnosis and pathophysiology. Clin Perinatol 22:281-342

28. Watterberg KL, Demers LM, Scott SM, Murphy S (1996) Chorioamnionitis and early lung inflammation in infants in whom bronchopulmonary dysplasia developes. Pediatrics 97: 210-215

29. Bry K, Lappalainen U, Hallman M (1997) Intra-amniotic interleukin-1 accelerates surfactant protein synthesis in fetal rabbits and improves lung stability after premature birth. J Clin Invest 99:2992-2999

30. Bry K, Lappalainen U, Hallman M (1997) Maturational effects of IL-1 on fetal lungs are dependent on route of cytokine administration. Pediatr Res 41:42

31. Emerson GA, Bry K, Hallman M et al (1997) Intra-amniotic interleukin-1 alpha treatment alters postnatal adaptation in premature lambs. Biol Neonate 72:370-379

32. Glumoff V, Väyrynen O, Kangas T, Hallman M (1999) Degree of lung maturity determines the direction of interleukin-1 induced effect on the expression of surfactant proteins. Am J Respir Cell Mol Biol (in press)

33. Leff JA, Baer JW, Bodman ME et al (1993) Interleukin-1-induced lung neutrophil accumulation and oxygen metabolite mediated lung leak in rats. J Appl Physiol 266:2-8

34. Repine JE (1994) Interleukin-1-mediated acute lung injury and tolerance to oxidative injury. Environ Health Prospect 102[Suppl 10]:75-78

35. Väyrynen O, Glumoff V, Kangas T, Hallman M (1999) Endotoxin-induced changes in expression of surfactant proteins are dependent on the degree of lung maturity. Pediatr Res 45:896

36. Glumoff V, Väyrynen O, Kangas T, Hallman M (1999) Expression of surfactant proteins. Interaction between interleukin-1 (IL-1) and dexamethasone (Dx). Pediatr Res 45:892

37. Aikio O, Vuopala K, Pokela M-L, Hallman M (1999) Diminished inducible nitric oxide synthase expression in fulminant early-onset neonatal pneumonia. Pediatrics (in press) 